\title{
Concurrent performance of two memory tasks: Evidence for domain-specific working memory systems
}

\author{
GIANNA COCCHINI, ROBERT H. LOGIE, SERGIO DELLA SALA, and SARAH E. MACPHERSON \\ University of Aberdeen, Aberdeen, Scotland \\ and \\ ALAN D. BADDELEY \\ University of Bristol, Bristol, England
}

\begin{abstract}
Previous studies of dual-task coordination in working memory have shown a lack of dual-task interference when a verbal memory task is combined with concurrent perceptuomotor tracking. Two experiments are reported in which participants were required to perform pairwise combinations of (1) a verbal memory task, a visual memory task, and perceptuomotor tracking (Experiment 1), and (2) pairwise combinations of the two memory tasks and articulatory suppression (Experiment 2). Tracking resulted in no disruption of the verbal memory preload over and above the impact of a delay in recall and showed only minimal disruption of the retention of the visual memory load. Performing an ongoing verbal memory task had virtually no impact on retention of a visual memory preload or vice versa, indicating that performing two demanding memory tasks results in little mutual interference.Experiment 2 also showed minimal disruption when the two memory tasks were combined, although verbal memory (but not visual memory) was clearly disrupted by articulatory suppression interpolated between presentation and recall. These data suggest that a multiple-component working memory model provides a better account for performance in concurrent immediate memory tasks than do theories that assume a single processing and storage system or a limited-capacity attentional system coupled with activated memory traces.
\end{abstract}

The ability of human beings to perform more than one thing at a time has long been a focus of study in the literature on human attention and memory. However, there is a continuing debate as to what determines success or failure in dual- or multiple-task performance. A number of studies have demonstrated that with some combinations of tasks, healthy adult participants can perform under demanding dual-task conditions with very little performance degradation on either task relative to single-task baseline levels (Baddeley, Bressi, Della Sala, Logie, \& Spinnler, 1991; Baddeley, Logie, Bressi, Della Sala, \& Spinnler, 1986; Della Sala, Baddeley, Papagno, \& Spinnler, 1995; Duff \& Logie, 2001; Greene, Hodges, \& Baddeley, 1995). In some studies, no dual-task decrement has been observed-for example, when a processing task is combined with a memory task (e.g., Duff \& Logie, 2001). In other studies, dualtask decrements have appeared when a tracking task was combined with a verbal memory task, but they were lim-

This research was supported by Grant 050793 from the Wellcome Trust and U.K. Medical Research Council Co-operative Component Grant G0000259 within MRC Co-operative Group Grant G9901359. G.C. is now at the University of East London. We are grateful to Fergus Craik and to Veronica Dark for their helpful comments on an earlier version of this manuscript. Correspondence should be addressed to R. H. Logie, Department of Psychology, University of Aberdeen, Aberdeen AB24 2UB, Scotland (e-mail: r.logie@abdn.ac.uk). ited to modest reductions in performance levels on the order of $10 \%-12 \%$ despite very high task demands for each of the tasks being combined (Baddeley et al., 1991; Baddeley et al., 1986; Logie, Zucco, \& Baddeley, 1990). This suggests that each task might employ different specialized cognitive functions that can operate largely in parallel.

The relative lack of dual-task disruption with particular task combinations has been associated with the operation of a multiple-component working memory system thought to offer online processing and temporary storage of information by means of a number of specialized cognitive functions (Baddeley, 1986; Baddeley \& Logie, 1999). Within this model, each of the tasks used by Baddeley et al. (1991; Baddeley et al., 1986) was interpreted as drawing on separate specialized resources respectively for visuospatial (tracking) and for verbal (digit span) processing and temporary storage. This interpretation has empirical support from a range of dual-task studies carried out with healthy adults, showing that it is the choice of the type of tasks that are combined, and not the overall cognitive demand of dual-task requirements, that determines whether or not performance will be substantially impaired under dualtask conditions (e.g., Farmer, Berman, \& Fletcher, 1986; Logie, Cocchini, Della Sala, \& Baddeley, 2002; Logie et al., 1990; Wickens \& Yeh, 1983; for a recent review, see Baddeley \& Logie, 1999). 
One contrasting approach, initially proposed by Broadbent (1958), characterizes cognitive resources as comprising a single yet flexible facility of limited capacity (Engle, Kane, \& Tuholski, 1999; Just \& Carpenter, 1992; Newell, 1990) that can accomplish both processing and temporary storage. As demands for processing or storage exceed the predetermined limit, the performance of an individual will deteriorate. This view suggests that dual-task performance is made possible by time sharing the tasks, and that performance of each task will be unimpaired as long as the resources required by the two concurrent tasks do not exceed the total resources available. One version of this model has been proposed by Just and Carpenter, who argue that processing and storage rely on a single but flexible working memory system, with greater demands on processing leading to less available resources for storage and vice versa. However, this account cannot readily explain the relatively modest dual-task effects found in healthy adults for some task combinations, given that these effects are small even when the processing and memory demands have been adjusted to ensure that each component task is being performed at a very challenging level (i.e., at span) for each individual participant (e.g., Baddeley et al., 1991; Baddeley et al., 1986; Duff \& Logie, 2001).

The multiple-componentworking memory system provides a viable framework within which to account for dual-task performance in healthy adults. However, a caveat should be noted for the selection of tasks used in the Baddeley et al. (1991; Baddeley et al., 1986) studies. One of the tasks focused on immediate, serial ordered, oral recall of digit sequences presented aurally. This was performed concurrently with perceptuomotor tracking of a randomly moving target. One possible reason for the relative lack of dual-task impairment with healthy participants is that the recall task relied on immediate verbal memory, whereas the tracking task had no memory component. As indicated earlier, the result cannot readily be explained within a general processing and resource model, given that, within such a model, both the memory tasks and the tracking task would draw on a single, general purpose, limited-capacity system. However, the results could be interpreted by assuming that there is a general purpose immediate memory system that holds the digits and that is distinct from a perceptuomotor system that is responsible for controlling tracking performance. This account would not require the assumption that there are domain-specific components of working memory and might pose difficulties for our previous interpretation of the very limited dual-task interference effects found in healthy adults.

The major aim of this paper is to address these concerns by investigating the effects on performance of combining two memory tasks rather than combining a memory task with a demand on perceptuomotor control. To this end we report two experiments. In Experiment 1, healthy young adults performed dual-task combinations of immediate memory for aurally presented digit sequences, and immediate memory for visually presented matrix patterns. We also combined each of the memory tasks with a percep- tuomotor tracking task. This was in part to replicate and extend the results of our previous procedure (Baddeley et al., 1991; Baddeley et al., 1986; Della Sala et al., 1995), which combined digit span with tracking. However, this additional manipulation was also to investigate whether any dual-task interference might arise because of reliance on overlapping, domain-specific resources that are part of a multiple-componentsystem, employed for both visual immediate memory and for tracking, but not for digit recall. In each case, task demand was adjusted according to the ability of the individual participants.

In combining two memory tasks within the present experimental aims, it was essential to ensure that any mutual disruption was not the consequence of competition for sensory input or response output channels-for example, having to listen to digits and watch a visual pattern simultaneously or being required to repeat back digit sequences while recalling a pattern of squares. Therefore, in the dual-task conditions, we adopted a preload procedure in which participants held in memory the material for one task while they encoded and recalled material for the other memory task.

The general load theory of cognition would predict dual-task decrements with all task combinations; in particular, combining two tasks that both require temporary memory resources should result in a substantial cost of concurrence. A multiple-resource theory would predict dual-task interference only when two concurrent tasks employ aspects of the same cognitive resource. Within the latter view, there should be very limited if any mutual interference when the two memory tasks are combined, because verbal temporary memory is seen as quite distinct from visuospatial temporary memory (e.g., Baddeley \& Logie, 1999). In contrast, we should expect mutual interference when combining digit recall with articulatory suppression (e.g., Levy, 1971). The prediction is less clear for combining visual pattern recall with tracking, given the evidence for a possible separation between visual temporary memory and a dynamic spatial system involved in directing movement (e.g., Della Sala, Gray, Baddeley, Allamano, \& Wilson, 1999; Logie, 1995; Logie, Engelkamp, Dehn, \& Rudkin, 2001; Logie \& Pearson, 1997; Pickering, Gathercole, Hall, \& Lloyd, 2001).

\section{EXPERIMENT 1}

\section{Method}

\section{Participants}

Participants were 30 university students, 15 females and 15 males. Their mean age was 21.93 years $(S D=3.48$, range $=20-35)$ and they had a mean of 15.9 years of education $(S D=1.30$, range $=$ 14-18). They were paid a modest honorarium for taking part.

\section{Tests}

Perceptuomotor tracking. For this test participants were shown on a computer screen a target comprising a red oval with dark spots about $2.5 \mathrm{~cm}$ long and $2 \mathrm{~cm}$ wide. The stimulus resembled an insect known as a "ladybug" in North America and known as a "ladybird" in the U.K. We will use the term ladybird to refer to the stimulus in the remainder of the paper. Participants were then given a lightsensitive stylus that was to be placed on the target, which then began to move randomly around the screen. The task was to keep the stylus 
placed on the moving target. The speed of the ladybird could be set at different levels. Speed Level 1 corresponded to a movement of the target of approximately $3.5 \mathrm{~cm}$ per second. The difference between one speed level and the next was about $1 \mathrm{~cm}$ per second. For example, Speed Level 2 was approximately $4.5 \mathrm{~cm}$ per second, whereas Speed Level 10 was about $12.5 \mathrm{~cm}$ per second. Since the speed of movement of the ladybird was sinusoidal, the speed at each level of difficulty was an average and not a constant speed. The ladybird remained red as long as the light pen was in contact, but changed to green when contact was lost, returning to red when contact was regained.

The monitor screen was placed at an angle of $30^{\circ}$ from horizontal, because we have found in previous studies that this angle is less physically tiring than using a vertical screen (Baddeley et al., 1991).

In the initial adaptive phase, the ladybird moved slowly (at Speed Level $2=4.5 \mathrm{~cm}$ per $\mathrm{sec}$ ). The speed level increased to the next level if, over a period of $5 \mathrm{sec}$, the participant maintained contact with the target for at least $60 \%$ of the time. If time on target was less than $40 \%$, the speed level was reduced to the next lower level. Finally, if time on target was between $40 \%$ and $60 \%$, the speed level did not change. When the speed level remained constant for $15 \mathrm{sec}$ (three 5sec periods), the adaptive tracking phase was complete, and this speed level was used as a measure of the tracking ability for the individual.

To avoid fatigue from a lengthy adaptive tracking phase, speed level changes at the lower level involved single steps from 1 to 5 , whereas higher speed levels involved changes of two steps at any given time. This also allowed for lower ability people to have a reasonable amount of practice, but at the same time higher ability people would not perform a dramatically larger number of trials, which would possibly result in substantially different levels of practice according to individual ability.

Serial digit recall. Participants listened to lists of digits from a tape; their task was to repeat the digits in serial order. The digits were read by a native English speaker at a rate of two per second.

Digit span was individually determined by first asking participants to repeat sequences of two digits. Following correct recall of two out of three sequences, the sequence length was incremented by one digit and the procedure continued. There were no restrictions in time for recall. Participants were presented with three sequences at each length, and testing ceased when the participant failed to recall at least two out of three sequences. Individual span was taken as the longest sequence at which the participant recalled at least two out of three sequences correctly.

Visual pattern recall. Participants were presented with paper black and white checkerboard patterns that were difficult to encode verbally (Della Sala et al., 1999). These were generated by filling (in black) half of the $2.5-\mathrm{cm}$ squares at random. Patterns that obviously formed canonical shapes such as letters or numbers were excluded.

Visual pattern span was individually determined by first presenting participants with a $2 \times 2$ pattern for $3 \mathrm{sec}$. Next, participants were given a blank $2 \times 2$ grid in which they had to mark with a cross the previously filled squares. There was no time limit for their responses. Following correct recall of one out of three patterns at any given level, complexity was increased by presenting a $2 \times 3$ matrix with new random patterns. This procedure continued by gradually increasing the number of squares in the matrix (e.g., $3 \times 3,3 \times 4,4 \times$ 4 , and so on) with three trials at each level of complexity until the participant failed to recall the filled squares on all three trials at a given level of complexity. The level of complexity of a pattern was defined as the number of filled squares in a grid, and ranged from a minimum of 2 to a maximum of 15 . The individual visual pattern span was taken as the mean of the last three patterns for which filled squares were correctly recalled.

\section{Procedure}

The experiment consisted of three phases.

Phase 1. First the presentation parameters were determined for each participant on the tracking task, the digit recall task, and the visual pattern recall task.
Phase 2. Next, single-task performance was measured on all three tasks, with the demands of each task set for each individual according to the presentation parameters determined in Phase 1 . The single-tracking task consisted of three trials lasting $15 \mathrm{sec}$ each. The first $3 \mathrm{sec}$ for each trial were considered as a "run-in" period and were not included in the analyses. This was intended to remove effects that might arise from initiation of performance, rather than from continuous performance of the tracking task.

Single-task recall performance for visual patterns and for digit sequences was measured under immediate recall and under delayed recall conditions for each task. Immediate recall required the participant to repeat the digit sequence or to recall the visual pattern immediately after presentation, and participants performed three immediate recall trials for each task. Each trial consisted of two digit sequences or two visual patterns. Each trial lasted between 15 and $20 \mathrm{sec}$ to allow for the variable presentation and recall times for the different spans across participants. For delayed recall, following presentation of a single-digit sequence, or a single pattern, there was a blank interval of $15 \mathrm{sec}$ during which participants were asked to rehearse mentally the sequence of digits or continually visualize the pattern. Each participant performed three delayed recall trials. The single-task delayed recall conditions were included to act as control conditions against which to measure the impact of holding the items as a preload under the dual-task conditions, described below. For each task, single-task immediate recall was completed before delayed recall for all participants. Participants who performed poorly on visual pattern recall, digit recall, or tracking (below $70 \%$ for the memory tests and below $40 \%$ for tracking) were excluded from the final analyses.

Phase 3. Finally, each participant performed pairs of tasks in concert. Dual-task performance was measured on each memory task, with a preload for one task followed by a 15 -sec delay that was filled with presentation and recall of material for the other memory task on a repeated cycle or filled with continuous tracking. At the end of the filled delay, the preload was to be recalled. This resulted in four conditions, illustrated in Figure 1. As in Phase 2, the first $3 \mathrm{sec}$ of tracking for each trial were excluded from the analyses.

Participants generally were able to complete two sequences or patterns (presentation and recall) for the interpolated task during the delay period. For each dual-task condition, participants performed six trials.

All participants performed Phase 1, followed by Phase 2 and then Phase 3, but within each phase the order of presentation of the three single tasks and the dual-task combinations was counterbalanced across participants.

\section{Results}

Percentage recall or tracking accuracy was calculated for each participant in each condition. The data from 2 participants were excluded from the final analysis because of poor performance (below 70\%) in single-task digit immediate recall. The final analyses were carried out on data from 28 participants. Differences among conditions were evaluated via analysis of variance (ANOVA).

\section{Digit Recall}

The mean proportion correct scores (and standard deviations) for each condition are shown in Table 1. There was a significant cost of delay in the single-task condition $\left[F(1,27)=13.05, M S_{\mathrm{e}}=83.6, p<.001\right]$. This was a surprising result given that participants were free to rehearse the digits during the delay. There was a significant effect of dual-task demand with immediate recall $[F(1,27)=$ $\left.8.68, M S_{\mathrm{e}}=82.7, p<.01\right]$, suggesting that maintaining a visual preload interfered with performance. An ANOVA 


\section{Digits + Patterns}

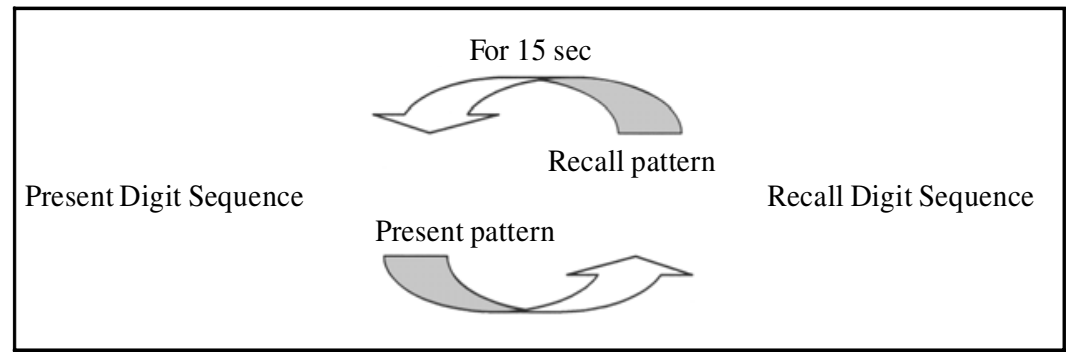

Digits + Tracking

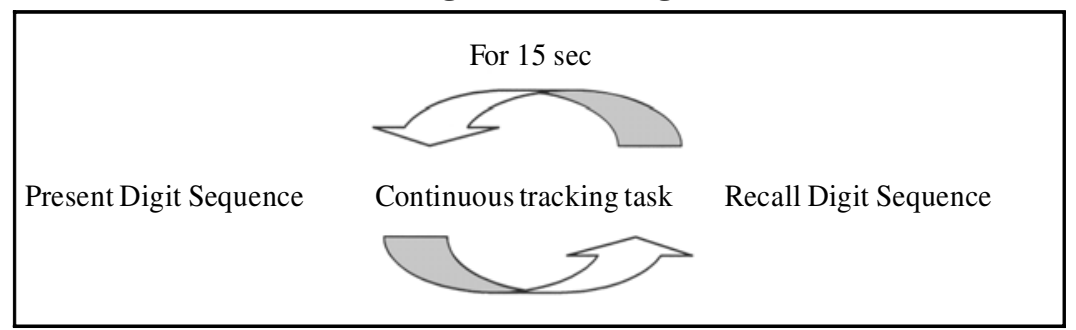

\section{Patterns + Digits}

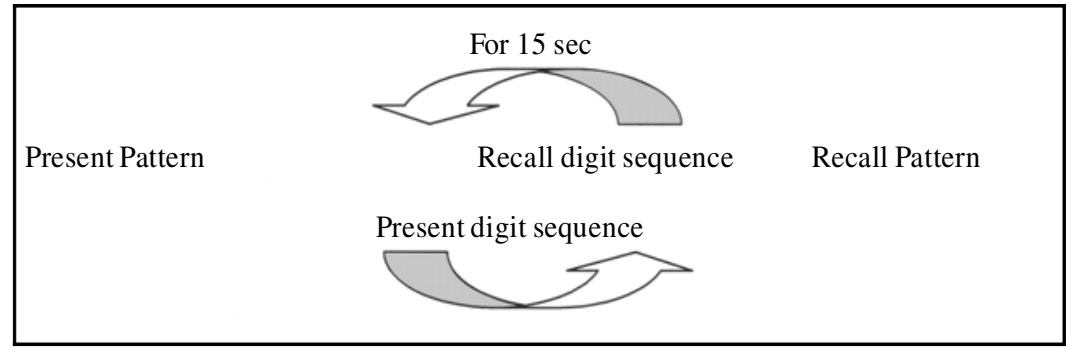

\section{Patterns + Tracking}

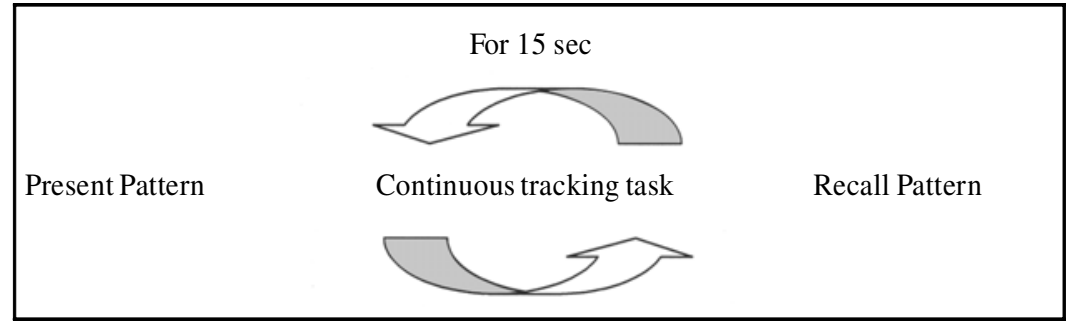

Figure 1. Summary of procedures for the four dual-task conditions used in Experiment 1.

comparing the three delayed digit recall conditions (single task, combined with the visual pattern task, and combined with the tracking task) showed no significant difference among the conditions $(F<1)$, indicating a lack of dualtask cost in delayed recall for either type of interpolated task.

\section{Visual Pattern Recall}

The mean proportion correct scores (and standard deviations) for each condition are shown in Table 1 . There was no effect of delay in the single-task condition $(F<1)$, indicating that participants were able to maintain the pattern without loss over the delay interval. There was a sig- nificant effect of dual-task demand with immediate recall $\left[F(1,27)=11.4, M S_{\mathrm{e}}=37.4, p<.01\right]$, suggesting that maintaining a digit preload interfered with performance. An ANOVA comparing the three delayed visual pattern recall conditions (single task, combined with the visual pattern task, and combined with the tracking task) showed a significant difference among conditions $[F(2,54)=9.5$, $\left.M S_{\mathrm{e}}=83.3, p<.001\right]$. Post hoc analyses with NewmanKeuls showed that visual pattern recall was impaired when combined with tracking $(p<.001)$ but not when combined with digit recall. Moreover, performance with tracking was significantly poorer than performance with digit recall $(p<.01)$. 
Table 1

Mean Proportion Correct on the Digit and Visual Pattern Memory Tasks as a Function of Time of Recall (Immediate or Delayed) and Type of Task (Single or Dual) in Experiment 1

\begin{tabular}{lccccc}
\hline & \multicolumn{2}{c}{ Digit } & & \multicolumn{2}{c}{ Visual Pattern } \\
\cline { 2 - 3 } \cline { 5 - 6 } Task & $M$ & $S D$ & & $M$ & $S D$ \\
\hline Immediate recall & & & & \\
$\quad$ Single task & 91.09 & 8.06 & & 89.89 & 5.16 \\
$\quad$ Dual task & 83.93 & 12.28 & & 84.38 & 7.78 \\
Delayed recall & & & & \\
$\quad$ Single task & 82.26 & 15.43 & & 89.72 & 9.43 \\
$\quad$ Dual task & & & & \\
$\quad$ With tracking & 85.11 & 11.43 & & 79.14 & 10.72 \\
$\quad$ With digit & - & - & & 85.52 & 8.23 \\
$\quad$ With pattern & 81.12 & 17.94 & & - \\
\hline
\end{tabular}

\section{Tracking Test}

An ANOVA on tracking task performance showed that there was no difference $(F<1)$ between percentage times on target for single-task tracking $(M=57.60, S D=5.73)$, for tracking with a digit preload $(M=56.06, S D=8.0)$, or for tracking with a pattern preload $(M=55.66, S D=$ 7.77). Note that the tracking task under dual-task conditions was always performed during the interpolated period.

\section{Discussion}

In our previous studies (Baddeley et al., 1991; Baddeley et al., 1986; Della Sala et al., 1995) we demonstrated that healthy adults can perform concurrently a demanding verbal memory task and a demanding perceptuomotor task with minimal impact on the performance of either task. We interpreted these findings as reflecting the operation of distinct, specialized cognitive resources for each task. Our concern was that the observed disruption in these previous studies was minimal simply because we were combining a task that loads temporary memory with a task that requires perceptuomotor control. This could point to a separation between working memory and the perceptuomotor system, but not necessarily to fractionation of working memory into specialized components. The experiment reported here demonstrated that combining two memory tasks does not yield consistent reductions in performance. Retention of a digit preload was not disrupted by an interpolated visual memory task, and retention of a visual pattern preload was not disrupted by interpolated digit recall. We also showed no significant disruption of a digit span preload by interpolated tracking.

Two significant dual-task effects did appear that were not predicted by a multiple-component working memory theory. However, both effects were rather small if we consider that the participants were asked to perform two very demanding tasks concurrently. Immediate recall of digits dropped by around $8 \%$ when a visual pattern preload was retained, and immediate recall of a visual pattern dropped by around $5 \%$ when a digit preload was retained. Although these effects were statistically significant, there was not the large drop in dual-task performance that might be predicted by a model that assumes a single, general purpose memory system. However, the modest dual-task interference observed might suggest some overlap in the cognitive resources required for both tasks under these conditions. It could also suggest an overlap in the forms of coding used for both tasks - for example, using some form of verbal labeling of the patterns to supplement retention of a decaying temporary visual memory trace while attempting to use subvocal rehearsal for retention of the digit sequences for immediate recall (see Logie, Della Sala, Laiacona, Chalmers, \& Wynn, 1996, for a discussion of coding strategies). However, this explanation is rather post hoc. Moreover, given the modest level of these dual-task effects, it would be important to establish whether they are robust before exploring possible accounts further. This issue is addressed in Experiment 2.

One further dual-task effect was the disruption of delayed pattern recall by interpolated perceptuomotor tracking, although again the disruption was relatively modest, with a drop of around $11 \%$. Previous studies have shown tracking to be disruptive of tasks that have a heavy spatial, dynamic component such as the Brooks (1967) matrix path recall (e.g., Baddeley \& Lieberman, 1980; Quinn \& Ralston, 1986) or the Corsi (Milner, 1972) block procedure (e.g., Smyth \& Scholey, 1994). Visual immediate memory tasks are largely unaffected by concurrent physical movement (e.g., Della Sala et al., 1999). Moreover, several studies have demonstrated that separate cognitive resources appear to support, respectively, dynamic spatial tasks and visual immediate memory tasks using developmental fractionation (Hitch, 1990; Logie \& Pearson, 1997), double dissociations in patients (e.g., Della Sala et al., 1999; Grossi, Becker, Smith, \& Trojano, 1993), and neuroanatomicalsegregation in neuroimaging studies (e.g., Courtney, Petit, Maisog, Ungerleider, \& Haxby, 1998; Smith, Jonides, Koeppe, Awh, Schumacher, \& Minoshima, 1995). This evidence has led to the suggestion of a temporary dynamic spatial memory system referred to as the "inner scribe" and a more passive visual temporary memory system referred to as the "visual cache" (Baddeley \& Logie, 1999; Logie, 1995). The modest interference found in the present data between visual matrix memory and tracking is consistent with this distinction, with tracking using the resources of the inner scribe and visual matrix memory relying on the visual cache. The tracking task is of course not a pure spatial dynamic task in that it involves visual input and monitoring as well as motor control and movement. This might account for why the dual-task interference of interpolated tracking on delayed pattern recall is so modest. It might also account for the complete lack of an effect on tracking performance of holding a visual pattern preload.

The experiment demonstrated that even two very demanding memory tasks can be performed concurrently with minimal interference. On the whole, these data speak to the possibility that working memory comprises a number of domain-specific memory systems. However, the results are open to the possible interpretation that items for the preload task are encoded in long-term memory while participants are performing the interpolated task. When 
participants are asked to recall the preload, this would be retrieved from what remains of the trace in long-term memory. Were this to be the case, we might expect that in the unfilled delay condition, participants would be free to rehearse the items in working memory. If, in the filled delay conditions, they were relying on long-term memory, then we might expect that recall of the preload would be poorer in the filled delay compared with the unfilled delay conditions. It is clear from our data that these conditions did not differ (Table 1). Nevertheless, it remains a possibility that participants rely on long-term memory in the unfilled delay condition as well. Indeed, the rather surprising effect on digit recall of an unfilled delay might be consistent with reliance on a decaying trace in long-term memory rather than the use of subvocal rehearsal during the delay. This invites a further empirical test to assess the extent to which working memory is involved in the retention of the preload material. Experiment 2 was intended to address this issue by assessing whether the digit recall preload was sensitive to disruption by interpolated articulatory suppression, a manipulation that has been shown repeatedly to disrupt the contents of verbal working memory (e.g., Baddeley, Lewis, \& Vallar, 1984; Levy, 1971; Murray, 1986). Thus Experiment 2 was similar to Experiment 1 except that articulatory suppression was employed instead of a perceptuomotor tracking task. Experiment 2 also involved combining the two memory tasks employed in Experiment 1 with the intention of assessing the reliability of the results from the concurrent memory manipulation.

\section{EXPERIMENT 2}

\section{Method}

\section{Participants}

Thirty university students ( 15 females and 15 males) between 19 and 33 years (mean age $=23.20$ years, $S D=3.65$ ) were recruited for this experiment. They had a mean education of 15.13 years $(S D=$ 2.19 , range $=12-19$ ). None of the participants had taken part in Experiment 1 , and they received a modest honorarium for participating.

\section{Tests}

The ordered digit recall and visual pattern recall tests of Experiment 1 were used in Experiment 2. The tracking task was replaced with an articulatory suppression task.

Articulatory suppression. Participants were asked to continuously repeat aloud the word $g o$ at a rate of two per second for $15 \mathrm{sec}$. The dependent variable was the rate of articulation, that is, the number of utterances per second. As for tracking in Experiment 1, the first $3 \mathrm{sec}$ of utterances were excluded from the analyses under both singleand dual-task conditions to avoid the possible impact of initiation rather than of continuous, concurrent articulation.

\section{Procedure}

In the present experiment, the same general procedure as in Experiment 1 was employed, except the perceptuomotor tracking task was replaced with an articulatory suppression task. The single articulatory suppression task consisted of three 15 -sec trials. As with the perceptuomotor tracking task, the articulatory suppression dual task was only performed as an intervening task during the 15-sec delay.

\section{Results}

Data analysis was similar to that reported in Experiment 1, except that the mean articulation rate was calculated for each participant under each condition, instead of tracking accuracy. One of the participants was excluded from the final analyses because performance was below $70 \%$ on single-task digit immediate recall. The final analyses were conducted on the data for the remaining 29 participants.

\section{Digit Recall Test}

The mean proportion correct scores (and standard deviations) for each condition are shown in Table 2. The unexpected finding of a cost to delay found in Experiment 1 was not replicated. There was no effect of delay in the single-task condition $(F<1)$. Table 2 indicates an effect of overall dual-task demand with immediate recall, but, unlike this effect in Experiment 1, this failed to reach significance $\left[F(1,28)=3.77, M S_{\mathrm{e}}=77.67, .05<p<0.1\right]$. An ANOVA comparing the three delayed digit recall conditions (unfilled delay, digit recall combined with the visual memory task, or with articulatory suppression) showed a significant difference $\left[F(2,56)=44.01, M S_{\mathrm{e}}=\right.$ $131.71, p<.0001]$. Newman-Keuls post hoc analyses demonstrated that digit span recall was impaired when combined with articulatory suppression and when combined with visual pattern recall. Digit span recall was significantly worse when combined with articulatory suppression than with visual pattern recall $(p<.001)$.

\section{Visual Pattern Recall}

The mean proportion correct scores (and standard deviations) for each condition are shown in Table 2, which indicates that there was a drop in performance following a delay in the single-task condition, but this failed to reach significance $\left[F(1.28)=3.01, M S_{\mathrm{e}}=75.45, .05<p<.1\right]$. As found in Experiment 1, there was a significant effect of dual-task demand with immediate recall $[F(1,28)=$ $\left.7.13, M S_{\mathrm{e}}=32.21, p<.05\right]$, indicating that immediate recall of patterns is affected by holding a digit preload. However, unlike Experiment 1, there was not a significant difference between the three delayed recall conditions $(F<1)$, namely unfilled delayed recall or delayed filled with digit recall or with articulatory suppression. This indicates that there was no effect of dual-task demand in delayed recall for either combination.

Table 2

Mean Proportion Correct on the Digit and Visual Pattern Memory Tasks as a Function of Time of Recall (Immediate or Delayed) and Type of Task (Single or Dual) in Experiment 2

\begin{tabular}{lccccc}
\hline & \multicolumn{2}{c}{ Digit } & & \multicolumn{2}{c}{ Visual Pattern } \\
\cline { 2 - 3 } \cline { 5 - 6 } Task & $M$ & $S D$ & & $M$ & $S D$ \\
\hline Immediate recall & & & & \\
$\quad$ Single task & 88.84 & 7.50 & & 91.07 & 6.07 \\
$\quad$ Dual task & 84.35 & 12.45 & 87.09 & 5.72 \\
Delayed Recall & & & & \\
$\quad$ Single task & 88.41 & 14.42 & 87.11 & 9.89 \\
$\quad \begin{array}{l}\text { Dual task } \\
\text { With suppression }\end{array}$ & 60.71 & 21.02 & & 87.57 & 6.56 \\
$\quad$ With digit & - & - & 85.29 & 10.15 \\
$\quad$ With pattern & 79.49 & 13.75 & & - & - \\
\hline
\end{tabular}




\section{Articulatory Suppression}

Responses with interresponse intervals of less than $300 \mathrm{msec}$ or greater than $1,500 \mathrm{msec}$ were excluded from the analyses, resulting in a loss of $4.47 \%$ of the responses. The mean rates of articulation (the number of utterances per second) were as follows: for articulatory suppression alone, $M=1.72(S D=0.17)$, with digit preload, $M=1.70$ $(S D=0.12)$, and with pattern preload, $M=1.71(S D=$ $0.17)$. The demands of dual-task did not affect the articulation rate $(F<1)$.

\section{Discussion}

The purpose of Experiment 2 was to assess whether participants rely on long-term memory to retain material presented as a preload, while performing a concurrent task. This was addressed by employing a secondary task (articulatory suppression) that has previously been shown to disrupt immediate verbal memory. The expectation is that if long-term memory is being used for retention of material in the delayed recall conditions, then we should find little or no disruption from articulatory suppression. The results demonstrated that delayed recall of a digit preload is disrupted by interpolated articulatory suppression in a fashion that is widely reported when the two tasks are performed concurrently. In the latter case, it is assumed that retention of the digit sequence relies on subvocal rehearsal within the phonological loop component of working memory, and repeating aloud an irrelevant word acts to disrupt the rehearsal function of this system (for reviews see Baddeley, 1986; Baddeley \& Logie, 1999). Therefore, our results suggest that the preload digits are being retained in the phonological loop of working memory.

The findings are consistent with those of previous studies of delayed recall of verbal material. For example, Baddeley and Hitch (1976) demonstrated that holding a sequence of six digits while performing a verbal reasoning task resulted in a reduction in performance. Studies using the classic Brown-Peterson (e.g., Brown, 1958) procedures involved a preload of letters or digits with a verbal interpolated task, such as counting backward, a manipulation that resulted in a reduction in digit recall compared with an unfilled delay.

There was a suggestion that delayed recall of digits was disrupted by interpolated pattern recall. However, the level of disruption was much less than that resulting from articulatory suppression. Holding a digit preload disrupted immediate recall of visual patterns, but the drop in performance, shown in Table 2, was very modest compared with the very substantial effect of articulatory suppression on delayed recall of the digits. There was no indication from the analysis of an impact on delayed recall of patterns of either of the dual-task demands. This indicates that the result obtained in Experiment 1 of a significant disruption of delayed pattern recall by a digit preload may not be robust.

The level of disruption observed for articulatory suppression is comparable with that reported previously for the effects of this manipulation on immediate serial or- dered recall (e.g., Baddeley et al., 1984; Baddeley, Thomson, \& Buchanan, 1975; Gregg, Freedman, \& Smith, 1989; Murray, 1986), suggesting that delayed recall over a period of $15 \mathrm{sec}$ in our experiments relies on rehearsal in working memory and requires no more involvement of long-term memory than that which possibly contributes to immediate recall. The level of the disruptive effects on immediate recall (in previous studies) and of delayed recall in Experiment 2 is in striking contrast with the very modest disruptive effects that we observed when combining a demanding immediate verbal memory load with a demanding visuospatial memory load. The specificity of the interference observed reinforces our conclusion from Experiment 1 that combining two memory tasks that rely on theoretically separate components of working memory has, at worst, a very small impact on the performance of each task.

\section{GENERAL DISCUSSION}

Taken together, the results from both experiments are difficult to reconcile with a single attentional or general purpose processing and storage resource being responsible for both tasks. The results are more compatible with a multiple-componentworking memory system comprising a number of domain-specific systems.

One possible caveat in regard to Experiment 1 arises from previous studies reporting evidence that a concurrent perceptuomotor task might interfere with verbal memory performance, although there is some debate as to whether such interference occurs during the encoding or retrieval phase (e.g., Fernandes \& Moscovitch, 2000; Naveh-Benjamin, Craik, Perretta, \& Tonev, 2000; NavehBenjamin \& Guez, 2000; Troyer, Winocur, Craik, \& Moscovitch, 1999). However, in none of these studies were the task demands adjusted according to the individual ability of the participants, and in studies where this has been implemented, the dual-task effects were either greatly reduced or absent (see, e.g., Belleville, Rouleau, \& Caza, 1998; Hartley \& Little, 1999). In Experiment 1, it appeared that immediate recall of digits showed a drop in performance when it was the interpolated task combined with recall of a pattern preload. Also, immediate pattern recall was impaired when combined with a digit preload. We offered one possible account based on the use of supplementary verbal coding to support delayed recall of a visual pattern. However, the disruptive effects were quite small, and they were not replicated in Experiment 2. The lack of replication suggests that they may not be robust effects, and that they might be prone to the impact of individual differences in strategies used by different participants across the two experiments (e.g., Logie et al., 1996).

It might be possible to account for the data reported in Experiment 1 within a modified version of the general attentional resources model recently proposed by Cowan $(1999,2000)$. In his view, there is a central, capacity-limited attentional system that can focus on one task at any one time. Short-term memory arises from temporary activa- 
tion of items in long-term memory, and this activation decays over a period of a few seconds, leading to a time limitation in addition to the attentional capacity limitation. In Experiment 1, participants were given memory preloads followed by a period in which they performed either a memory task or perceptuomotor tracking. Cowan might argue that the memory preload involves temporary activation that gradually decays while the attentional system is focused on the interpolated task. During delayed recall, attention is then focused on retrieving items from decaying traces, hence the observed impact of delayed recall in our own data, in the absence of substantive impact on memory for the interpolated task. Cowan further argues that the amount of activation at any one time is apparently unlimited. This would account for the lack of substantial mutual interference when our participants held a digit preload while encoding and retrieving visual patterns and vice versa.

However, Cowan's $(1999,2000)$ model runs into some difficulty accounting for the indications of differential interference obtained in Experiments 1 and 2, and in a range of previous studies (e.g., Della Sala et al., 1999; Farmer et al., 1986; Logie et al., 1990; Pickering, 2001; Quinn \& Ralston, 1986). This difficulty becomes more acute in interpreting findings showing greater interference when a movement task is combined with a visuospatial memory task than when it is performed with verbal recall, even although there is no reason to expect that motor control would involve codes that overlap with those used for retaining a visuospatial pattern. However, the multiple-resource model referred to in the introduction makes explicit why a domain-specific system for visuo-spatial tasks might have a role in enactment of physical movement (see Logie, 1995; Logie et al., 2001). Moreover, there is strong neuropsychologicalevidence for highly selective domain-specific deficits resulting from focal lesions (see, e.g., Della Sala \& Logie, 1993), and the specificity of these deficits would be very difficult to explain in terms of coding similarity without recourse to a link between domain-specific codes and domain-specific cognitive systems.

One further aspect of Cowan's $(1999,2000)$ argument is that interference effects arise from similarities among the material or among the memory codes involved in the two tasks (e.g., Sanders \& Schroots, 1969). However, often similarity may be determined only post hoc with the interpretation arising from the data pattern rather than a theoretically motivated prediction. In Experiment 1, the matrix patterns bore no resemblance to the ladybird target used for the tracking task. Moreover, any single, limited attentional capacity model, including Cowan's, would meet a significant challenge when attempting to account for a lack of dual-task interference when two very demanding tasks are performed simultaneously. This was the case in our previous studies of tracking and memory (Baddeley et al., 1991; Baddeley et al., 1986) and in a wide range of other published reports (for reviews, see Baddeley \& Logie, 1999; Logie, 1995).
The dual-task findings are more readily explained by a multiple-resource model, with each resource functioning more or less independently and with demands on one resource having little impact on the efficiency of other resources. Given that two of the tasks that we have used focus on temporary retention, their performance may be supported by domain-specific temporary memory systems that can act in concert with little mutual interference. This approach is much less prone to the accusation levied above at the coding similarity concept that interference patterns are interpreted post hoc. Specifically, each of the putative memory resources has arisen from a wide range of data patterns, drawing on not just differences in codes employed and specific dual-task interference, but also on double dissociations observed in the patterns of impairment and sparing following contrasting forms of brain damage (e.g., Farah, Hammond, Levine, \& Calvanio, 1988; Shallice \& Warrington, 1970) and clearly dissociable patterns of brain activation in the healthy brain associated with performance of tasks linked with theoretically dissociable components of cognition (e.g., Awh et al., 1996; Smith \& Jonides, 1997). The multiple-resources model can encompass accounts for all of these sources of data and makes predictions as to the kinds of tasks that would employ each form of resource. An account based on differences or similarities among codes used to support task performance, even if it were not post hoc, has much more limited explanatory utility. Its explanatory and predictive value might be enhanced if the different forms of code were to be associated with the operation of, respectively, different networks in the brain, or with different cognitive systems. However, it would then become indistinguishable from an account based on multiple resources. It therefore offers, at best, no more convincing an explanation than does a multiple-resource model of the dual-task interference effects, or lack of such effects, that we have observed.

The multiple-resource approach does raise the issue of how the operation of these independent resources might be coordinated. One approach is to consider coordination as an emergent property of the function of the interfaces and the communication flow between the independent systems (e.g., Barnard, 1999). However, this leads to a model of some complexity that has been implemented computationally, but that may be difficult to falsify and has yet to prove to be a fruitful framework within which to generate experiments. An alternative is to draw on the hypothesis from the working memory framework that the central executive provides this coordination function. This role for the central executive was an element of the original Baddeley and Hitch (1974) conception of working memory. However, precisely what role a coordination function might play, and what specific tasks it performs, remains unclear.

One possible role for a coordination function is that it implements encoding and retrieval of material that is held within each of the specialized memory resources (Duff \& Logie, 2001). In the single-task condition, the encoding 
and retrieval processes have to deal only with one kind of material and one type of memory resource (e.g., a verbal buffer such as the phonological loop). In dual-task conditions such as those in the present experiment, verbal material would be held in a verbal buffer, and visual material would be held in a visual buffer (such as the visual cacheLogie, 1995). The coordinating mechanism would perform encoding for one buffer, then encoding and retrieval from another buffer for the interpolated memory task, followed by retrieval from the first buffer. From our previous studies, if a memory task is concurrent with another attention-demanding task (such as tracking), the encoding and retrieval operations for the memory task might be somewhat impaired by tracking, but the memory retention itself would not be. In both scenarios (preload and concurrent dual task), performance would depend on the efficiency of both the storage mechanisms and the coordination mechanism. Dual-task conditions would place no greater demands on each of the memory systems, but would have an impact on the efficiency of the coordination device. Therefore the requirement to swap between buffers or swap between encoding/retrieval while following a randomly moving target might lead to a modest drop in performance such as those that we have observed. This offers a possible account for some of our data; investigation of the coordination function in dual-task performance is the subject of a separate but related series of experiments reported elsewhere (Baddeley et al., 1991; Baddeley et al., 1986; Logie et al., 2002).

In conclusion, the debate as to the nature of the cognitive functions involved in concurrent performance of the tasks that we have described may yet find a resolution in an alternative model that offers the most coherent explanation for the widest range of data sets, while still generating testable hypotheses. Thus far, a multiple-component system that incorporates specialized memory functions appears to offer the best account for the findings of both the present experiments and related previous research.

\section{REFERENCES}

Awh, A, Jonides, J., Smith, E. E, Schumacher, E. H., Koeppe, R. A. \& KATz, S. (1996). Dissociation of storage and rehearsal in verbal working memory: Evidence from positron emission tomography. Psychological Science, 7, 25-31.

BADDElEy, A. D. (1986). Working memory. Oxford: Oxford University Press.

Baddeley, A. D., Bressi, S., Della Sala, S., Logie, R. H., \& SpinnLER, H. (1991). The decline of working memory in Alzheimer's disease: A longitudinal study. Brain, 114, 2521-2542.

BADDELEY, A. D., \& Hitch, G. J. (1974). Working memory. In G. H. Bower (Ed.), The psychology of learning and motivation (Vol. 8, pp. 47-90). New York: Academic Press.

BADDELEy A. D., \& HITCH, G. J. (1976). Verbal reasoning and working memory. Quarterly Journal of Experimental Psychology, 28, 603-621.

BADDEley, A. D., Lewis, V. J., \& VAllaR, G. (1984). Exploring the articulatory loop. Quarterly Journal of Experimental Psychology, 36, 233-252.

Baddeley, A. D., \& Lieberman, K. (1980). Spatial working memory. In R. S. Nickerson (Ed.), Attention and performance VIII (pp. 521539). Hillsdale, NJ: Erlbaum.

BAdDeley, A. D., \& Logie, R. H. (1999). Working memory: The multiple component model. In A. Miyake \& P. Shah (Eds.). Models of working memory (pp. 28-61). New York: Cambridge University Press.
Baddeley, A. D., Logie, R., Bressi, S., Della Sala, S., \& Spinnler, H. (1986). Senile dementia and working memory. Quarterly Journal of Experimental Psychology, 38A, 603-618.

Baddeley, A. D., Thomson, N., \& Buchanan, M. (1975). Word length and the structure of short-term memory. Journal of Verbal Learning \& Verbal Behavior, 14, 575-589.

BARnARD, P. J. (1999). Interacting cognitive subsystems: Modelling working memory phenomena within a multi-processor architecture. In A. Miyake \& P. Shah (Eds.), Models of working memory (pp. 298339). New York: Cambridge University Press.

Belleville,S., Rouleau, N., \& CaZA, N. (1998). Effect of normal aging on the manipulation of information in working memory. Memory \& Cognition, 26, 572-583.

Broadbent, D. E. (1958). Perception and communication. New York: Pergamon.

BrooKs, L. R. (1967). The suppression of visualization by reading. Quarterly Journal of Experimental Psychology, 19, 289-299.

BRown, J. (1958). Some tests of the decay theory of immediate memory. Quarterly Journal of Experimental Psychology, 10, 12-21.

Courtney, S. M., Petit, L., Maisog, J. M., Ungerleider, L. G., \& HАХву, J. V. (1998). An area specialized for spatial working memory in human frontal cortex. Science, 279, 1347-1351.

Cowan, N. (1999). An embedded-processes model of working memory. In A. Miyake \& P. Shah (Eds.), Models of working memory (pp. 62101). New York: Cambridge University Press.

Cowan, N. (2000). The magical number 4 in short-term memory: A reconsideration of mental storage capacity. Behavioral \& Brain Sciences, 24, 87-114.

Della Sala, S., Baddeley, A., Papagno, C., \& Sinnler, H. (1995). Dual-task paradigm. A means to examine the central executive. In J. Grafman, K. J. Holyoak, \& F. Boller (Eds.), Structure and functions of the human prefrontal cortex (Annals of the New York Academy of Sciences, Vol. 769, pp.161-190). New York: New York Academy of Sciences.

Della Sala, S., Gray, C., Baddeley, A., Allamano, N., \& Wilson, L. (1999). Pattern span: A tool for unwelding visuo-spatial memory. Neuropsychologia, 37, 1189-1199.

Della Sala, S., \& Logie, R. (1993). When working memory does not work: The role of working memory in neuropsychology. In F. Boller \& H. Spinnler (Eds.), Handbook of neuropsychology (Vol 8. pp. 1-63). Amsterdam: Elsevier.

Duff, S., \& Logie, R. H. (2001). Processing and storage in working memory: Cognitive capacity or multiple components? Quarterly Journal of Experimental Psychology, 54A, 31-48.

Engle, R. W., Kane, M. J., \& Tuholski, S. W. (1999). Individual differences in working memory capacity and what they tell us about controlled attention, general fluid intelligence, and functions of the prefrontal cortex. In A. Miyake \& P. Shah (Eds.), Models of working memory (pp.102-134). New York: Cambridge University Press.

Farah, M. J., Hammond, K. M., Levine, D. N., \& Calvanio, R. (1988). Visual and spatial mental imagery: Dissociable systems of representation. Cognitive Psychology, 20, 439-462.

Farmer, E. W., Berman, J. V. F., \& Fletcher, Y. L. (1986). Evidence for a visuo-spatial scratch-pad in working memory. Quarterly Journal of Experimental Psychology, 38A, 675-688.

Fernandes, M. A., \& Moscovitch, M. (2000). Divided attention and memory: Evidence of substantial interference effects at retrieval and encoding. Journal of Experimental Psychology: General, 129, 155-176.

Greene, J., Hodges, J., \& BAdDELey, A. D. (1995). Autobiographical memory and executive functions in early dementia of Alzheimer type. Neuropsychologia, 33, 1647-1670.

Gregg, V. H., Freedman, C. M., \& Smith, D. K. (1989). Word frequency, articulatory suppression and memory span. British Journal of Psychology, 80, 363-374.

Grossi, D., Becker., J. T., Smith, C., \& Trojano, L. (1993). Memory for visuospatial patterns in Alzheimer's disease. Psychological Medicine, 23, 65-70.

Hartley, A. A., \& Little, D. M. (1999). Age-related differences and similarities in dual-task interference. Journal of Experimental Psychology: General, 128, 416-449.

Hгтch, G. J. (1990). Developmental fractionation of working memory. In G. Vallar \& T. Shallice (Eds.), Neuropsychological impairments of 
short-term memory (pp. 221-246).Cambridge: Cambridge University Press.

Just, M., \& CARPenter, P. (1992). A capacity theory of comprehension: Individual differences in working memory. Psychological Review, 99, 122-149.

LEvy, B. A. (1971). The role of articulation in auditory and visual shortterm memory. Journal of Verbal Learning \& Verbal Behavior, 10, 123-132.

LogIE, R. H. (1995). Visuo-spatialworking memory. Hove, U.K.: Erlbaum.

Logie, R. H., Cocchini, G., Della Sala, S., \& Baddeley, A. D. (2002). Is there a specific executive capacity for dual-task co-ordination? Evidence from Alzheimer's Disease. Manuscript submitted for publication.

Logie, R. H., Della Sala, S., Laiacona, M., Chalmers, P., \& Wynn, V. (1996). Group aggregates and individual reliability: The case of verbal short-term memory. Memory \& Cognition, 24, 305-321.

Logie, R. H., EngelKamp, J., Dehn, D., \& Rudkin, S. (2001). Actions, mental actions, and working memory. In M. Denis, R. H. Logie, C. Cornoldi, J. Engelkamp, \& M. De Vega (Eds.), Imagery, language and visuo-spatial thinking (pp. 161-183). Hove, U.K.: Psychology Press.

Logie, R H., \& Pearson, D. G. (1997). The inner eye and the inner scribe of visuo-spatial working memory: Evidence from developmental fractionation. European Journal of Cognitive Psychology, 9, 241-257.

Logie, R. H., Zucco, G., \& BAdDeley, A. D. (1990). Interference with visual short-term memory. Acta Psychologica, 75, 55-74.

Milner, B. (1972). Disorders of learning and memory after temporal lobe lesions in man. Clinical Neurosurgery, 19, 421-446.

MuRRAY, D. J. (1986). Level of articulation and short-term recognition following brief probe delays. Bulletin of the Psychonomic Society, 24, 103-106.

Naveh-Benjamin, M., Craik, F. I. M., Perretta, J. G., \& Tonev, S. T. (2000). The effects of divided attention on encoding and retrieval processes: The resiliency of retrieval processes. Quarterly Journal of Experimental Psychology, 53A, 609-625.

Naveh-Benjamin, M., \& GUEZ, J. (2000). Effects of divided attention on encoding and retrieval processes: Assessment of attentional costs and a componential analysis. Journal of Experimental Psychology: Learning, Memory, \& Cognition, 26, 1461-1482.

NeWELL, K. M. (1990). Unified theories of cognition: The 1987 William James Lectures. Cambridge, MA: Harvard University Press.

Pickering, S. (2001). Cognitive approaches to fractionation of visuospatial working memory. Cortex, 37, 457-473.

Pickering, S., Gathercole, S., Hall, M., \& Lloyd, S. (2001). Development of memory for pattern and path: Further evidence for the fractionation of visuo-spatial memory. Quarterly Journal of Experimental Psychology, 54A, 397-420.

Quinn, J. G., \& RALston, G. E. (1986). Movement and attention in visual working memory. Quarterly Journal of Experimental Psychology, 38A, 689-703.

Sanders, A. F., \& Schroots, J. J. F. (1969). Cognitive categories and memory span III: Effects of similarity on recall. Quarterly Journal of Experimental Psychology, 21, 21-28.

Shallice, T., \& Warrington, E. K. (1970). Independent functioning of verbal memory stores: A neuropsychological study. Quarterly Journal of Experimental Psychology, 22, 261-273.

Sмith, E. E., \& Jonides, J. (1997). Working memory: A view from neuroimaging. Cognitive Psychology, 33, 5-42.

Smith, E. E., Jonides, J., Koeppe, R A., Awh, E., Schumacher, E. H., \& Minoshima, S. (1995). Spatial versus object working memory: PET investigations. Journal of Cognitive Neuroscience, 7, 337-356.

Smyth, M. M., \& Scholey, K. A. (1994). Interference in immediate spatial memory. Memory \& Cognition, 22, 1-13.

Troyer, A. K., Winocur, G., Craik, F. I. M., \& Moscovitch, M. (1999). Source memory and divided attention: Reciprocal costs to primary and secondary tasks. Neuropsychology, 13, 467-474.

Wickens, C. D., \& YEH, Y. Y. (1983). The dissociation between subjective workload and performance: A multiple resources approach. In L. Haugh \& A. Pope (Eds.), Proceedings of the 27th Annual Meeting of the Human Factors Society. Santa Monica, CA: Human Factors Press.

(Manuscript received September 13, 2001; revision accepted for publication June 6, 2002.) 